\title{
Separable recursive gradient algorithm for dynamical systems based on the impulse response signals
}

\author{
Ling Xu*, Feng Ding*, and Erfu Yang
}

\begin{abstract}
The identification for process control systems is considered in this paper based on the impulse response signals from the discrete measurements. By taking advantage of impulse signals and through the model parameter decomposition, two dependent identification models are constructed and two identification sub-algorithms are presented based on the nonlinear gradient optimization. In terms of the associated items of the parameters to be estimated between two derived sub-algorithms, a separable recursive gradient parameter estimation method is proposed by designing an interactive and recursive estimation. The performance tests and the comparison experiments are carried out by the simulation examples.
\end{abstract}

Keywords: Parameter estimation; System response; Gradient search; Impulse signal; Recursive algorithm

\section{INTRODUCTION}

The dynamical behavior of systems can be obtained from the measurements of the input and output of systems. Therefore, the selection of excitation signals is an important step in the design of the identification experiments. On the other hand, the identification results can be significantly influenced by the input signals which are applied to the systems to be identified [1-5]. In terms of identification experiments, sinusoidal waves and combinational sine signals, step signals, impulse signals and pseudo-random binary sequences are the most frequently used test signals. The systems to be identified are excited by these excitation signals and will generate the system responses, which contain the important dynamical information. Through the discrete observations of the system responses, we can develop identification methods to construct the models of the systems. Generally, the impulse signal is the simplest and easy to realize in many applications among the excitation signals. Some identification methods are obtained based on special input signal excitations [6-8].

There is an ordinary phenomenon that some models show the features of the combination of linear and nonlinear relations, in which one can separate the system parameters into two parts: a linear part and a nonlinear part [9]. In system identification, the separable technique is adopted to decompose the identification models into several submodels to reduce the complexity $[10,11]$. Many identification methods have been proposed in terms of linear systems or nonlinear systems by means of the model decomposition or parameter decomposition [12-14] and can be applied to different fields [15-18].
Many nonlinear optimization can be solved by separable nonlinear least squares method, in which a model can be denoted by a combination of linear and nonlinear functions. Recently, these models are used widely in a variety of applications such as neural networks, time-series analysis, signal analysis and other fields [19]. For the problems of separable nonlinear least squares, the separated parameter sets are dependent on each other. For this problem, the general solution is to optimize all the parameters of the systems regardless of the features of parameters which cannot show the preponderance of the separable methods. Meanwhile, that the parameter decomposition gives rise to a new difficulty is how to solve the associated items among the separated sub-algorithms. It is worth noting that many identification approaches are obtained by experiment techniques [20,21]. Many parameter estimation methods have been developed for linear systems [22,23] and nonlinear systems [24,25] and can be applied to many areas [26-31] for constructing their mathematical models.

The following Section 2 introduces the identification problem and the characteristic of identification models. Section 3 derives a recursive gradient sub-algorithm in terms of the the linear parameters. Section 4 presents a recursive gradient sub-algorithm for the nonlinear parameters. Section 5 proposes a separable recursive gradient algorithm by uniting two sub-algorithms. Section 6 puts forward an integral recursive gradient algorithm for comparing the performance between the separable method and the non-separable method. Section 7 provides an example and takes the Monte Carlo experiment and comparison test to illustrate the effectiveness of the proposed separable identification method. Section 8 is the conclusions of

This work was supported by the National Natural Science Foundation of China (No. 61873111) and the 111 Project (B12018).

Ling Xu and Feng Ding are with the Key Laboratory of Advanced Process Control for Light Industry (Ministry of Education), School of Internet of Things Engineering, Jiangnan University, Wuxi 214122, PR China (e-mails: lingxu0848@163.com, fding@jiangnan.edu.cn). Erfu Yang is with the Department of Design, Manufacture and Engineering Management, Space Mechatronic Systems Technology Laboratory, Strathclyde Space Institute, University of Strathclyde, Glasgow G1 1XJ, Scotland, United Kingdom (e-mail: erfu.yang@strath.ac.uk).

* Corresponding author: School of Internet of Things Engineering, Jiangnan University, Wuxi 214122, PR China. 
this paper.

\section{PROBLEM ANALYSIS}

The transfer function models are of popularity for describing linear time-invariant systems. In general, the transfer function model for the linear time-invariant system with $n$ order takes the following form:

$$
G(s)=g(s, \vartheta)=\frac{C(s)}{D(s)},
$$

where $C(s)$ and $D(s)$ are the numerator polynomial and denominator polynomial, i.e.,

$$
\begin{aligned}
& C(s)=c_{m} s^{m}+c_{m-1} s^{m-1}+\cdots+c_{1} s+c_{0}, \\
& D(s)=d_{n} s^{n}+d_{n-1} s^{n-1}+\cdots+d_{1} s+d_{0}, \quad d_{n} \neq 0 .
\end{aligned}
$$

The notations are illustrated as follows. $\vartheta$ is the parameter vector which contains the system parameters to be identified, i.e., $d_{n}, d_{n-1}, \cdots, d_{0}, c_{m}, c_{m-1}, \cdots, c_{0} . m$ is the order of the numerator polynomial. $n$ is the order of the denominator polynomial, and $n>m$.

Under the condition that the system only has distinct poles and a unit impulse excitation, the impulse response takes the following mathematical form:

$$
y(t)=f(a, b, t)=\sum_{i=1}^{n} b_{i} \mathrm{e}^{-a_{i} t}+v(t) .
$$

where $a:=\left[a_{1}, a_{2}, \cdots, a_{n}\right] \in \mathbb{R}^{n}$ and $b:=\left[b_{1}, b_{2}, \cdots, b_{n}\right]$ are the unknown parameter vectors, $t$ is the time variable and $v(t)$ is the observation noise.

From the above description, we find that even though the system is linear time-invariant, the system response is a highly nonlinear function. Moreover, the unknown parameters $a_{i}$ and $b_{i}$ are comprised in the response function. Therefore, one can acquire the information of the system by collecting the discrete measurements from the system response signals. Then, the problem of the parameter estimation is converted into an optimal problem by building a cost function regarding to the unknown parameters.

Here is a special characteristic that $y(t)$ is a linear function about the parameters $b_{i}$ and $y(t)$ is a nonlinear function about the parameters $a_{i}$. This issue motivates us to separate the parameters into two parameter vectors to be identified. One is the linear parameter vector $b:=$ $\left[b_{1}, b_{2}, \cdots, b_{n}\right]^{\mathrm{T}} \in \mathbb{R}^{n}$, the other is the nonlinear parameter vector $a:=\left[a_{1}, a_{2}, \cdots, a_{n}\right]^{\mathrm{T}} \in \mathbb{R}^{n}$. For the purpose of gaining enough real-time information of the systems, we employ the dynamical data with increasing data length so as to obtain the whole process data and use these data dynamically.

Let the initial sampled moment be $t_{1}$. Then, the measurements from the initial moment $t_{1}$ to the current moment $t_{k}$ are described as $y\left(t_{1}\right), y\left(t_{2}\right), \cdots, y\left(t_{k}\right)$. With the increasing of $t_{k}$ over time, the length of the collected data also increases. Based on the dynamical observations with the increasing data length, define the objective function

$$
J(a, b):=\frac{1}{2} \sum_{j=1}^{k}\left[y\left(t_{j}\right)-f\left(a, b, t_{j}\right)\right]^{2} .
$$

From $J(a, b)$, it can be seen that the criterion function with incremental data length continuously absorbs new observed data. Therefore, more data can be involved in the recursive computation. Based on the separated parameter vectors $a$ and $b$, the objective function is separated into two sub-objective functions:

$$
\begin{aligned}
& J(a):=\frac{1}{2} \sum_{j=1}^{k}\left[y\left(t_{j}\right)-f\left(a, b, t_{j}\right)\right]^{2}, \\
& J(b):=\frac{1}{2} \sum_{j=1}^{k}\left[y\left(t_{j}\right)-\varphi_{b}\left(a, t_{j}\right) b\right]^{2} .
\end{aligned}
$$

where $\varphi_{b}\left(a, t_{j}\right)=\left[\mathrm{e}^{-a_{1} t_{j}}, \mathrm{e}^{-a_{2} t_{j}}, \cdots, \mathrm{e}^{-a_{n} t_{j}}\right]^{\mathrm{T}} \in \mathbb{R}^{n}$. As a result, two sub-algorithms can be derived by optimizing the above two separated criterion functions $J(a)$ and $J(b)$ separately. The deriving process is described in the following section.

\section{THE RECURSIVE GRADIENT SUB-ALGORITHM FOR LINEAR PARAMETERS}

Let us make a hypothesis that the nonlinear parameter vector $a$ is known and the linear parameter vector $b$ is unknown. Under this hypothesis, the aim of identification is to obtain the parameter estimate of the linear parameter vector $b$.

Define the information vector

$$
\varphi_{b}\left(a, t_{k}\right)=\left[\mathrm{e}^{-a_{1} t_{k}}, \mathrm{e}^{-a_{2} t_{k}}, \cdots, \mathrm{e}^{-a_{n} t_{k}}\right]^{\mathrm{T}} \in \mathbb{R}^{n} .
$$

Define the error between the observed output and model output:

$$
v\left(a, b, t_{k}\right):=y\left(t_{k}\right)-\sum_{i=1}^{n} b_{i} \mathrm{e}^{-a_{i} t_{k}} \in \mathbb{R} .
$$

Then, $v\left(a, b, t_{k}\right)$ can be expressed as

$$
v\left(a, b, t_{k}\right)=y\left(t_{k}\right)-\varphi_{b}^{\mathrm{T}}\left(a, t_{k}\right) b .
$$

Finding the search direction by taking the first-order derivative of the objective function $J(b)$ gives the gradient vector:

$$
\operatorname{grad}[J(b)]:=\frac{\partial J(b)}{\partial b}=-\sum_{j=1}^{k} \varphi_{b}\left(a, t_{j}\right) v\left(a, b, t_{j}\right) \in \mathbb{R}^{n} .
$$

Because the measurements are stacked with the time increasing, we define the stacked output vector $Y\left(t_{k}\right)$ and the stacked information matrix $\Phi_{b}\left(a, t_{k}\right)$ as

$$
\begin{aligned}
Y\left(t_{k}\right) & :=\left[\begin{array}{c}
y\left(t_{1}\right) \\
y\left(t_{2}\right) \\
\vdots \\
y\left(t_{k}\right)
\end{array}\right] \in \mathbb{R}^{k}, \\
\Phi_{b}\left(a, t_{k}\right):= & {\left[\begin{array}{c}
\varphi_{b}^{\mathrm{T}}\left(a, t_{1}\right) \\
\varphi_{b}^{\mathrm{T}}\left(a, t_{2}\right) \\
\vdots \\
\varphi_{b}^{\mathrm{T}}\left(a, t_{k}\right)
\end{array}\right] \in \mathbb{R}^{k \times n} . }
\end{aligned}
$$


As a result, the gradient vector $\operatorname{grad}[J(b)]$ becomes

$$
\begin{aligned}
\operatorname{grad}[J(b)] & =-\Phi_{b}^{\mathrm{T}}\left(a, t_{k}\right)\left[Y\left(t_{k}\right)-\Phi_{b}\left(a, t_{k}\right) b\right] \\
& =-\left[\Phi_{b}^{\mathrm{T}}\left(a, t_{k}\right) Y\left(t_{k}\right)-\Phi_{b}^{\mathrm{T}}\left(a, t_{k}\right) \Phi_{b}\left(a, t_{k}\right) b\right] \\
& =-\left[\xi_{b}\left(t_{k}\right)-R_{b}\left(t_{k}\right) b\right],
\end{aligned}
$$

where

$$
\begin{aligned}
\xi_{b}\left(t_{k}\right) & :=\Phi_{b}^{\mathrm{T}}\left(a, t_{k}\right) Y\left(t_{k}\right) \\
& =\xi_{b}\left(t_{k-1}\right)+\varphi_{b}\left(a, t_{k}\right) y\left(t_{k}\right) \in \mathbb{R}^{n}, \\
R_{b}\left(t_{k}\right) & :=\Phi_{b}^{\mathrm{T}}\left(a, t_{k}\right) \Phi_{b}\left(a, t_{k}\right) \\
& =R_{b}\left(t_{k-1}\right)+\varphi_{b}\left(a, t_{k}\right) \varphi_{b}^{\mathrm{T}}\left(a, t_{k}\right) \in \mathbb{R}^{n \times n} .
\end{aligned}
$$

The estimate of the linear parameter vector $b$ at $t_{k}$ is denoted by $\hat{b}\left(t_{k}\right):=\left[\hat{b}_{1}\left(t_{k}\right), \hat{b}_{2}\left(t_{k}\right), \cdots, \hat{b}_{n}\left(t_{k}\right)\right]^{\mathrm{T}}$. By means of the negative gradient search, the recursive gradient subalgorithm for identifying the linear parameter vector $b$ is developed as follows:

$$
\begin{aligned}
\hat{b}\left(t_{k}\right) & =\hat{b}\left(t_{k-1}\right)-\frac{1}{r_{b}\left(t_{k}\right)} \operatorname{grad}\left[J\left(\hat{b}\left(t_{k-1}\right)\right)\right] \\
& =\hat{b}\left(t_{k-1}\right)+\frac{1}{r_{b}\left(t_{k}\right)}\left[\xi_{b}\left(t_{k}\right)-R_{b}\left(t_{k}\right) \hat{b}\left(t_{k-1}\right)\right] \\
r_{b}\left(t_{k}\right) & =r_{b}\left(t_{k-1}\right)+\left\|\varphi_{b}\left(a, t_{k}\right)\right\|^{2} \\
\xi_{b}\left(t_{k}\right) & =\xi_{b}\left(t_{k-1}\right)+\varphi_{b}\left(a, t_{k}\right) y\left(t_{k}\right) \\
R_{b}\left(t_{k}\right) & =R_{b}\left(t_{k-1}\right)+\varphi_{b}\left(a, t_{k}\right) \varphi_{b}^{\mathrm{T}}\left(a, t_{k}\right) \\
\varphi_{b}\left(a, t_{k}\right) & =\left[\mathrm{e}^{-a_{1} t_{k}}, \mathrm{e}^{-a_{2} t_{k}}, \cdots, \mathrm{e}^{-a_{n} t_{k}}\right]^{\mathrm{T}} \\
\hat{b}\left(t_{k}\right) & =\left[\hat{b}_{1}\left(t_{k}\right), \hat{b}_{2}\left(t_{k}\right), \cdots, \hat{b}_{n}\left(t_{k}\right)\right]^{\mathrm{T}}
\end{aligned}
$$

Remark 1: The recursive sub-algorithm in (2)-(7) only can be used for estimating the linear parameter vector $b$ when the nonlinear parameter vector $a$ is known. If the nonlinear parameter vector $a$ is unknown, the recursive sub-algorithm (2)-(7) is in vain because there contains the unknown nonlinear parameter vector $a$ in the information vector $\varphi_{b}\left(a, t_{k}\right)$.

\section{THE RECURSIVE GRADIENT SUB-ALGORITHM FOR NONLINEAR PARAMETER VECTOR}

Based on the separable parameters, here derives the recursive gradient sub-algorithm for the nonlinear parameter vector $a$. Suppose that the linear parameter vector $b$ is known and the nonlinear parameter vector $a$ is unknown.

Taking the first-order derivative of the separated objective function $J(a)$ obtains the search direction, i.e., the gradient of $J(a)$ :

$$
\begin{aligned}
\operatorname{grad}[J(a)] & :=\frac{\partial J(a)}{\partial a} \\
& =\left[\frac{\partial J(a)}{\partial a_{1}}, \frac{\partial J(a)}{\partial a_{2}}, \cdots, \frac{\partial J(a)}{\partial a_{n}}\right]^{\mathrm{T}} \in \mathbb{R}^{n}, \\
\frac{\partial J(a)}{\partial a_{l}} & =\sum_{j=1}^{k} b_{l} t_{j} \mathrm{e}^{-a_{l} t_{j}} v\left(a, b, t_{j}\right), \quad l=1,2, \cdots, n, \\
v\left(a, b, t_{j}\right) & =y\left(t_{k}\right)-\sum_{i=1}^{n} b_{i} \mathrm{e}^{-a_{i} t_{j}} .
\end{aligned}
$$

The information vector is given by

$$
\begin{aligned}
\varphi_{a}\left(a, b, t_{k}\right):= & {\left[b_{1} t_{k} \mathrm{e}^{-a_{1} t_{k}}, b_{2} t_{k} \mathrm{e}^{-a_{2} t_{k}}, \cdots, b_{n} t_{k} \mathrm{e}^{-a_{n} t_{k}}\right]^{\mathrm{T}} } \\
& \in \mathbb{R}^{n} .
\end{aligned}
$$

The stacked information matrix is given by

$$
\Phi_{a}\left(a, b, t_{k}\right):=\left[\begin{array}{c}
\varphi_{a}^{\mathrm{T}}\left(a, b, t_{1}\right) \\
\varphi_{a}^{\mathrm{T}}\left(a, b, t_{2}\right) \\
\vdots \\
\varphi_{a}^{\mathrm{T}}\left(a, b, t_{k}\right)
\end{array}\right] \in \mathbb{R}^{k \times n} .
$$

Define the model output at $t_{k}$ as $f\left(a, b, t_{k}\right):=\sum_{i=1}^{n} b_{i} \mathrm{e}^{-a_{i} t_{k}} \in$ $\mathbb{R}$. The stacked model output vector is defined as

$$
F\left(a, b, t_{k}\right):=\left[\begin{array}{c}
f\left(a, b, t_{1}\right) \\
f\left(a, b, t_{2}\right) \\
\vdots \\
f\left(a, b, t_{k}\right)
\end{array}\right] \in \mathbb{R}^{k} .
$$

As a result, the gradient vector $\operatorname{grad}[J(a)]$ is expressed as

$$
\begin{aligned}
\operatorname{grad}[J(a)] & =\Phi_{a}^{\mathrm{T}}\left(a, b, t_{k}\right)\left[Y\left(t_{k}\right)-F\left(a, b, t_{k}\right)\right] \\
& =\Phi_{a}^{\mathrm{T}}\left(a, b, t_{k}\right) Y\left(t_{k}\right)-\Phi_{a}^{\mathrm{T}}\left(a, b, t_{k}\right) F\left(a, b, t_{k}\right) .
\end{aligned}
$$

Define the following recursive relationships:

$$
\begin{aligned}
\xi_{a}\left(a, b, t_{k}\right):= & \Phi_{a}^{\mathrm{T}}\left(a, b, t_{k}\right) Y\left(t_{k}\right) \\
= & \Phi_{a}^{\mathrm{T}}\left(a, b, t_{k-1}\right) Y\left(t_{k-1}\right) \\
& +\varphi_{a}\left(a, b, t_{k}\right) y\left(t_{k}\right) \in \mathbb{R}^{n}, \\
\zeta_{a}\left(a, b, t_{k}\right):= & \Phi_{a}^{\mathrm{T}}\left(a, b, t_{k}\right) F\left(a, b, t_{k}\right) \\
= & \Phi_{a}^{\mathrm{T}}\left(a, b, t_{k-1}\right) F\left(a, b, t_{k-1}\right) \\
& +\varphi_{a}\left(a, b, t_{k}\right) f\left(t_{k}\right) \in \mathbb{R}^{n} .
\end{aligned}
$$

Thus, the gradient vector $\operatorname{grad}[J(a)]$ can be expressed as

$$
\operatorname{grad}[J(a)]=\xi_{a}\left(a, b, t_{k}\right)-\zeta_{a}\left(a, b, t_{k}\right) .
$$

Denote $\hat{a}\left(t_{k}\right)=\left[\hat{a}_{1}\left(t_{k}\right), \hat{a}_{2}\left(t_{k}\right), \cdots, \hat{a}_{n}\left(t_{k}\right)\right]^{\mathrm{T}} \in \mathbb{R}^{n}$, where $\hat{a}\left(t_{k}\right)$ is the recursive estimate of the nonlinear parameter vector $a$ at $t_{k}$. According to the theory of the negative gradient search and optimizing the objective function $J(a)$, the recursive gradient sub-algorithm for estimating the nonlinear parameter vector $a$ is summarized as follows:

$$
\begin{aligned}
\hat{a}\left(t_{k}\right) & =\hat{a}\left(t_{k-1}\right)-\frac{1}{r_{a}\left(t_{k}\right)} \operatorname{grad}\left[J\left(\hat{a}\left(t_{k-1}\right), b\right)\right] \\
& =\hat{a}\left(t_{k-1}\right)-\frac{1}{r_{a}\left(t_{k}\right)}\left[\hat{\xi}_{a}\left(t_{k}\right)-\hat{\zeta}_{a}\left(t_{k}\right)\right] \\
r_{a}\left(t_{k}\right) & =r_{a}\left(t_{k-1}\right)+\left\|\varphi_{a}\left(\hat{a}\left(t_{k-1}\right), b, t_{k}\right)\right\|^{2} \\
\hat{\xi}_{a}\left(t_{k}\right) & =\xi_{a}\left(\hat{a}\left(t_{k-1}\right), b, t_{k}\right) \\
& =\hat{\xi}_{a}\left(t_{k-1}\right)+\varphi_{a}\left(\hat{a}\left(t_{k-1}\right), b, t_{k}\right) y\left(t_{k}\right), \\
\hat{\zeta}_{a}\left(t_{k}\right) & =\zeta_{a}\left(\hat{a}\left(t_{k-1}\right), b, t_{k}\right) \\
& =\hat{\zeta}_{a}\left(t_{k-1}\right)+\varphi_{a}\left(\hat{a}\left(t_{k-1}\right), b, t_{k}\right) \hat{f}_{a}\left(t_{k}\right), \\
\hat{\varphi}_{a}\left(t_{k}\right) & =\varphi_{a}\left(\hat{a}\left(t_{k-1}\right), b, t_{k}\right) \\
& =\left[b_{1} t_{k} \mathrm{e}^{\hat{a}_{1}\left(t_{k-1}\right) t_{k}}, b_{2} t_{k} \mathrm{e}^{\hat{a}_{2}\left(t_{k-1}\right) t_{k}}, \cdots, b_{n} t_{k} \mathrm{e}^{\left.\hat{a}_{n}\left(t_{k-1}\right) t_{k}\right]^{\mathrm{T}}},\right. \\
\hat{f}_{a}\left(t_{k}\right) & =f\left(\hat{a}\left(t_{k-1}\right), b, t_{k}\right)=\sum_{i=1}^{n} b_{i} \mathrm{e}^{\hat{a}_{i}\left(t_{k-1}\right) t_{k}}, \\
\hat{a}\left(t_{k}\right) & =\left[\hat{a}_{1}\left(t_{k}\right), \hat{a}_{2}\left(t_{k}\right), \cdots, \hat{a}_{n}\left(t_{k}\right)\right]^{\mathrm{T}} .
\end{aligned}
$$


Remark 2: From the presented recursive gradient subalgorithm in (8)-(14), we notice that the algorithm only can be used for determining the estimate of the nonlinear parameter vector $a$ in the condition that the linear parameter vector $b$ is known. Because the unknown parameters $b$ exist in the information vector $\hat{\varphi}_{a}\left(t_{k}\right)$, the recursive gradient sub-algorithm in (8)-(13) cannot be realized. This is the same problem as the sub-algorithm in (2)-(7).

\section{THE SEPARABLE RECURSIVE GRADIENT ALGORITHM}

In order to solve the problem which the sub-algorithms are unavailable, we combine the sub-algorithm in (2)-(7) for estimating the linear parameter vector $b$ and the subalgorithm in (8)-(14) for estimating the nonlinear parameter vector $a$. The key difficulty of the unavailable subalgorithms is that there are the associated unknown terms between the sub-algorithms. Thus the following measures are taken:

1) use $\hat{a}\left(t_{k-1}\right)$ to replace $a$ in (2)-(7);

2) use $\hat{b}\left(t_{k-1}\right)$ to replace $b$ in (8)-(14);

$3)$ estimate the separable parameter vectors $a$ and $b$ by interactive estimation technique. Then we obtain the separable recursive gradient (SRG) algorithm as follows:

$$
\begin{aligned}
& \hat{a}\left(t_{k}\right)=\hat{a}\left(t_{k-1}\right)-\frac{1}{r_{a}\left(t_{k}\right)} \operatorname{grad}\left[J\left(\hat{a}\left(t_{k-1}\right)\right]\right. \\
& =\hat{a}\left(t_{k-1}\right)-\frac{1}{r_{a}\left(t_{k}\right)}\left[\hat{\xi}_{a}\left(t_{k}\right)-\hat{\zeta}_{a}\left(t_{k}\right)\right], \\
& r_{a}\left(t_{k}\right)=r_{a}\left(t_{k-1}\right)+\left\|\hat{\varphi}_{a}\left(t_{k}\right)\right\|^{2}, \\
& \hat{\xi}_{a}\left(t_{k}\right)=\xi_{a}\left(\hat{a}\left(t_{k-1}\right), \hat{b}\left(t_{k-1}\right), t_{k}\right) \\
& =\hat{\xi}_{a}\left(t_{k-1}\right)+\hat{\varphi}_{a}\left(t_{k}\right) y\left(t_{k}\right), \\
& \hat{\zeta}_{a}\left(t_{k}\right)=\zeta_{a}\left(\hat{a}\left(t_{k-1}\right), \hat{b}\left(t_{k-1}\right), t_{k}\right) \\
& =\hat{\zeta}_{a}\left(t_{k-1}\right)+\hat{\varphi}_{a}\left(t_{k}\right) \hat{f}\left(t_{k}\right), \\
& \hat{\varphi}_{a}\left(t_{k}\right)=\varphi_{a}\left(\hat{a}\left(t_{k-1}\right), \hat{b}\left(t_{k-1}\right), t_{k}\right) \\
& =\left[\hat{b}_{1}\left(t_{k-1}\right) t_{k} \mathrm{e}^{\hat{a}_{1}\left(t_{k-1}\right) t_{k}}, \hat{b}_{2}\left(t_{k-1}\right) t_{k} \mathrm{e}^{\hat{a}_{2}\left(t_{k-1}\right) t_{k}}, \cdots,\right. \\
& \left.\hat{b}_{n}\left(t_{k-1}\right) t_{k} \mathrm{e}^{\hat{a}_{n}\left(t_{k-1}\right) t_{k}}\right]^{\mathrm{T}}, \\
& \hat{f}\left(t_{k}\right)=f\left(\hat{a}\left(t_{k-1}\right), \hat{b}\left(t_{k-1}\right), t_{k}\right) \\
& =\sum_{i=1}^{n} \hat{b}_{i}\left(t_{k-1}\right) \mathrm{e}^{\hat{a}_{i}\left(t_{k-1}\right) t_{k}}, \\
& \hat{b}\left(t_{k}\right)=\hat{b}\left(t_{k-1}\right)-\frac{1}{r_{b}\left(t_{k}\right)} \operatorname{grad}\left[J\left(\hat{b}\left(t_{k-1}\right)\right)\right] \\
& =\hat{b}\left(t_{k-1}\right)+\frac{1}{r_{b}\left(t_{k}\right)}\left[\hat{\xi}_{b}\left(t_{k}\right)-R_{b}\left(t_{k}\right) \hat{b}\left(t_{k-1}\right)\right], \\
& r_{b}\left(t_{k}\right)=r_{b}\left(t_{k-1}\right)+\left\|\varphi_{b}\left(\hat{a}\left(t_{k-1}\right), t_{k}\right)\right\|^{2}, \\
& \hat{\xi}_{b}\left(t_{k}\right)=\hat{\xi}_{b}\left(t_{k-1}\right)+\varphi_{b}\left(\hat{a}\left(t_{k-1}\right), t_{k}\right) y\left(t_{k}\right) \text {, } \\
& R_{b}\left(t_{k}\right)=R_{b}\left(t_{k-1}\right)+\varphi_{b}\left(\hat{a}\left(t_{k-1}\right), t_{k}\right) \varphi_{b}^{\mathrm{T}}\left(\hat{a}\left(t_{k-1}\right), t_{k}\right) \\
& \hat{\varphi}_{b}\left(t_{k}\right)=\varphi_{b}\left(\hat{a}\left(t_{k-1}\right), t_{k}\right) \\
& =\left[\mathrm{e}^{-\hat{a}_{1}\left(t_{k-1}\right) t_{k}}, \mathrm{e}^{-\hat{a}_{2}\left(t_{k-1}\right) t_{k}}, \cdots, \mathrm{e}^{-\hat{a}_{n}\left(t_{k-1}\right) t_{k}}\right]^{\mathrm{T}} \\
& \hat{a}\left(t_{k}\right)=\left[\hat{a}_{1}\left(t_{k}\right), \hat{a}_{2}\left(t_{k}\right), \cdots, \hat{a}_{n}\left(t_{k}\right)\right]^{\mathrm{T}}, \\
& \hat{b}\left(t_{k}\right)=\left[\hat{b}_{1}\left(t_{k}\right), \hat{b}_{2}\left(t_{k}\right), \cdots, \hat{b}_{n}\left(t_{k}\right)\right]^{\mathrm{T}} .
\end{aligned}
$$

In accordance with the above algorithm in (15)-(27), we summarize the computational steps for computing the parameter estimation vectors $\hat{a}\left(t_{k}\right)$ and $\hat{b}\left(t_{k}\right)$ of the system which is studied in this work.

1) Initiation: Let $k=1$, set $\hat{a}\left(t_{0}\right)=\left[\hat{a}_{1}\left(t_{0}\right), \hat{a}_{2}\left(t_{0}\right), \cdots\right.$, $\left.\hat{a}_{n}\left(t_{0}\right)\right]^{\mathrm{T}}, \hat{b}\left(t_{0}\right)=\left[\hat{b}_{1}\left(t_{0}\right), \hat{b}_{2}\left(t_{0}\right), \cdots, \hat{b}_{n}\left(t_{0}\right)\right]^{\mathrm{T}}, r_{a}\left(t_{0}\right)=1$, $r_{b}\left(t_{0}\right)=1, \hat{\xi}_{a}\left(t_{0}\right)=\mathbf{0}, \hat{\zeta}_{a}\left(t_{0}\right)=\mathbf{0}, \hat{\xi}_{b}\left(t_{0}\right)=\mathbf{0}, R_{b}\left(t_{0}\right)=\mathbf{0}$, and a recursive length $k_{\max }$.

2) Collect the impulse response data $y\left(t_{k}\right)$.

3) Calculate and construct the information vector $\hat{\varphi}_{a}\left(t_{k}\right)$ via (19), calculate the model output $\hat{f}\left(t_{k}\right)$ via (20) and calculate and construct the information vector $\hat{\varphi}_{b}\left(t_{k}\right)$ via (25).

4) Calculate $r_{a}\left(t_{k}\right)$ via (16), calculate vector $\hat{\xi}_{a}\left(t_{k}\right)$ via (17), calculate vector $\hat{\zeta}_{a}\left(t_{k}\right)$ via (18) and refresh the parameter estimation vector $\hat{a}\left(t_{k}\right)$ via (15).

5) Calculate $r_{b}\left(t_{k}\right)$ via (22), calculate vector $\hat{\xi}_{b}\left(t_{k}\right)$ via (23), calculate the matrix $R_{b}\left(t_{k}\right)$ via (24) and refresh the parameter estimation vector $\hat{b}\left(t_{k}\right)$ via (21).

6) Acquire the nonlinear parameter estimate $\hat{a}_{i}\left(t_{k}\right)$ from the vector $\hat{a}\left(t_{k}\right)$ in (26) and the linear parameter estimate $\hat{b}_{i}\left(t_{k}\right)$ from vector $\hat{b}\left(t_{k}\right)$ in $(27), i=1,2, \cdots, n$.

7) If recursion $k$ satisfies $k<k_{\max }$, then $k:=k+1$ and go to Step (2); otherwise obtain the parameter estimation vectors $\hat{a}\left(t_{k}\right)$ and $\hat{b}\left(t_{k}\right)$ and terminate the computational process.

\section{INTEGRAL RECURSIVE GRADIENT ALGORITHM}

For comparing the performance of the proposed separable algorithm, the integral recursive gradient algorithm is provided directly, in which the parameters to be identified are not separated. The recursive gradient (RG) algorithm is as follows:

$$
\begin{aligned}
& \hat{\theta}\left(t_{k}\right)=\hat{\theta}\left(t_{k-1}\right)+\frac{1}{r\left(t_{k}\right)} \\
& \times\left[\xi\left(\hat{\theta}\left(t_{k-1}\right), t_{k}\right)-\zeta\left(\hat{\theta}\left(t_{k-1}\right), t_{k}\right)\right] \\
& =\hat{\theta}\left(t_{k-1}\right)+\frac{1}{r\left(t_{k}\right)}\left[\hat{\xi}\left(t_{k}\right)-\hat{\zeta}\left(t_{k}\right)\right] \\
& r\left(t_{k}\right)=\operatorname{tr}\left[\Phi^{\mathrm{T}}\left(\hat{\boldsymbol{\theta}}\left(t_{k-1}\right), t_{k}\right) \Phi\left(\hat{\boldsymbol{\theta}}\left(t_{k-1}\right), t_{k}\right)\right] \\
& =r\left(t_{k-1}\right)+\left\|\varphi\left(\hat{\theta}\left(t_{k-1}\right), t_{k}\right)\right\|^{2} \\
& =r\left(t_{k-1}\right)+\left\|\hat{\varphi}\left(t_{k}\right)\right\|^{2}, \quad r\left(t_{0}\right)=1, \\
& \hat{\xi}\left(t_{k}\right):=\xi\left(\hat{\theta}\left(t_{k-1}\right), t_{k}\right) \\
& =\xi\left(\hat{\theta}\left(t_{k-1}\right), t_{k-1}\right)+\varphi\left(\hat{\theta}\left(t_{k-1}\right), t_{k}\right) y\left(t_{k}\right) \\
& =\hat{\xi}\left(t_{k-1}\right)+\hat{\varphi}\left(t_{k}\right) y\left(t_{k}\right), \\
& \hat{\zeta}\left(t_{k}\right):=\zeta\left(\hat{\theta}\left(t_{k-1}\right), t_{k}\right) \\
& =\zeta\left(\hat{\theta}\left(t_{k-1}\right), t_{k-1}\right)+\varphi\left(\hat{\theta}\left(t_{k-1}\right), t_{k}\right) f\left(\hat{\theta}\left(t_{k-1}\right), t_{k}\right) \\
& =\hat{\zeta}\left(t_{k-1}\right)+\hat{\varphi}\left(t_{k}\right) \hat{f}\left(t_{k}\right), \\
& \hat{\varphi}\left(t_{k}\right):=\varphi\left(\hat{\theta}\left(t_{k-1}\right), t_{k}\right) \\
& =\left[-\hat{b}_{1}\left(t_{k-1}\right) t_{k} \mathrm{e}^{-\hat{a}_{1}\left(t_{k-1}\right) t_{k}}, \mathrm{e}^{-\hat{a}_{1}\left(t_{k-1}\right) t_{k}},\right. \\
& -\hat{b}_{2}\left(t_{k-1}\right) t_{k} \mathrm{e}^{-\hat{a}_{2}\left(t_{k-1}\right) t_{k}}, \mathrm{e}^{-\hat{a}_{2}\left(t_{k-1}\right) t_{k}}, \cdots, \\
& \left.-\hat{b}_{n}\left(t_{k-1}\right) t_{k} \mathrm{e}^{-\hat{a}_{n}\left(t_{k-1}\right) t_{k}}, \mathrm{e}^{-\hat{a}_{n}\left(t_{k-1}\right) t_{k}}\right]^{\mathrm{T}}, \\
& \hat{f}\left(t_{k}\right)=f\left(\hat{\theta}\left(t_{k-1}\right), t_{k}\right) \\
& =\sum_{i=1}^{n} \hat{b}_{i}\left(t_{k-1}\right) \mathrm{e}^{-\hat{a}_{i}\left(t_{k-1}\right) t_{k}}, \\
& \hat{\boldsymbol{\theta}}\left(t_{k}\right)=\left[\hat{a}_{1}\left(t_{k}\right), \cdots, \hat{a}_{n}\left(t_{k}\right), \cdots, \hat{b}_{1}\left(t_{k}\right), \cdots, \hat{b}_{n}\left(t_{k}\right)\right]^{\mathrm{T}} \cdot(
\end{aligned}
$$


In accordance with the algorithm (28)-(34), we summarize the computational steps for estimating the parameter estimation vector $\hat{\theta}\left(t_{k}\right)$ as follows.

1) Initiation: Let $k=1$, set $\hat{\boldsymbol{\theta}}\left(t_{0}\right)=\left[\hat{a}_{1}\left(t_{0}\right), \hat{a}_{2}\left(t_{0}\right), \cdots\right.$, $\left.\hat{a}_{n}\left(t_{0}\right), \hat{b}_{1}\left(t_{0}\right), \hat{b}_{2}\left(t_{0}\right), \cdots, \hat{b}_{n}\left(t_{0}\right)\right]^{\mathrm{T}}, r\left(t_{0}\right)=1, \hat{\xi}\left(t_{0}\right)=\mathbf{0}$, $\hat{\zeta}\left(t_{0}\right)=\mathbf{0}$, and set the recursive step $k_{\max }$.

2) Collect the impulse response data $y\left(t_{k}\right)$.

3) Calculate and construct the information vector $\hat{\varphi}\left(t_{k}\right)$ via (32), and calculate the model output $\hat{f}\left(t_{k}\right)$ via (33).

4) Calculate $r\left(t_{k}\right)$ via (29), calculate vector $\hat{\xi}\left(t_{k}\right)$ via (30), and calculate vector $\hat{\zeta}\left(t_{k}\right)$ via (31)

5) Refresh the parameter estimation vector $\hat{\theta}\left(t_{k}\right)$ via (28).

6) If recursion $k$ satisfies $k<k_{\max }$, then $k:=k+1$ and go to Step (2); otherwise obtain the parameter estimates $\hat{\boldsymbol{\theta}}\left(t_{k}\right)$ from (34) and terminate the computational process.

The methods proposed in this paper can combine some mathematical tools [32-37] and strategies [38-41] to study the parameter estimation problems of different systems with colored noises and can be applied to other engineering systems [42-46] such as information systems [47-50] and networked systems [51-56] and so on.

\section{ILLUSTRATED EXAMPLE}

Consider the following nonlinear function

$$
y(t)=b_{1} \mathrm{e}^{-a_{1} t}+b_{2} \mathrm{e}^{-a_{2} t}+b_{3} \mathrm{e}^{-a_{3} t},
$$

where the true parameters of the system are $a_{1}=5.4$, $a_{2}=4.3, a_{3}=3.2, b_{1}=1, b_{2}=2$ and $b_{3}=1.8$. Its Laplace transform gives the transfer function $G(s)=$ $\frac{1}{s+5.4}+\frac{2}{s+4.3}+\frac{1.8}{s+3.2} \cdot y(t)$ is the unit impulse response of $G(s)$. In this section, the following simulation experiments are taken and the simulation results are analyzed.

\subsection{Monte Carlo experiment}

In this subsection, the Monte Carlo simulation tests using the measured data under different circumstance are taken to test the effectiveness of the proposed separable identification method. The experimental conditions are set as follows: 1) the noise variance is $\sigma^{2}=0.10^{2} ; 2$ ) the number of the Monte Carlo simulation tests is $20 ; 3$ ) the recursive step is $k_{\max }=200, k_{\max }=500, k_{\max }=1000$ and $k_{\max }=1500$, respectively; 4 ) the estimation error is computed according to the following equation:

$$
\delta\left(t_{k}\right):=\frac{\sqrt{\left\|\hat{a}\left(t_{k}\right)-a\right\|^{2}+\left\|\hat{b}\left(t_{k}\right)-b\right\|^{2}}}{\|a\|+\|b\|} .
$$

The parameter estimates obtained by the Monte Carlo tests are shown in Table 1, where SRG means the separable recursive gradient method. The estimation errors under different recursive steps for 20 Monte Carlo tests are drawn in Figure 1.

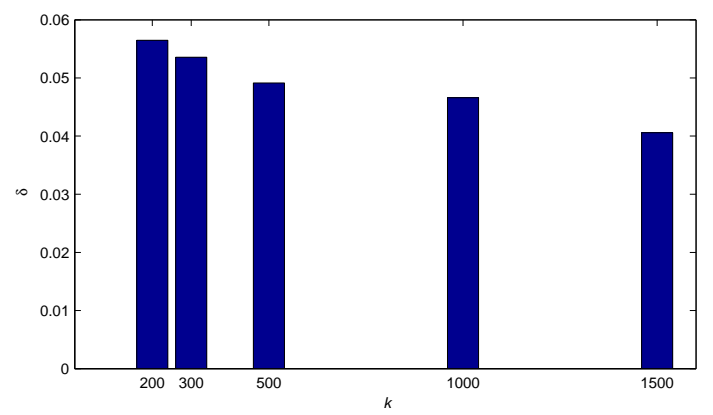

Fig. 1. The estimation errors under different recursive steps

\subsection{Performance comparison}

The performance comparison with other algorithms is an important way to illustrate the features of presented methods. In this subsection, the performance comparison experiment is implemented between the separable recursive gradient algorithm and the recursive gradient algorithm without separation. Firstly, we use the separable recursive gradient algorithm and the recursive gradient algorithm without separation to identify the parameters of the system in this example. Furthermore, different signalto-noise ratio (SNR) is considered because the noisy observations are involved in this example, where the SNR is defined as the ratio between the variance of signal and the variance of noise. Here, the experimental conditions are set as follows: 1) the sample period is $0.1 \mathrm{~s} ; 2$ ) the recursive step is $k_{\max }=2000 ; 3$ ) the estimation error is computed according to the following equation:

$$
\delta\left(t_{k}\right):=\frac{\sqrt{\left\|\hat{a}\left(t_{k}\right)-a\right\|^{2}+\left\|\hat{b}\left(t_{k}\right)-b\right\|^{2}}}{\|a\|+\|b\|} .
$$

Under the noisy measurement scenario, the performance of the proposed separable recursive gradient algorithm and the recursive gradient algorithm without separation are verified based on different SNRs. In the simulation, the SNR is set as 26.17 and 1.04. The estimated values of the system parameters and their estimation errors on different SNRs are displayed in Tables 2-3. The parameter estimation errors versus $k$ obtained via the separable recursive gradient method are shown in Figures 2 and 3 respectively. The parameter estimates obtained by these two methods under different SNRs and the original system parameters are illustrated in Figures 4 and 5 respectively.

In Figure 4, the parameter estimate of $a_{1}$ is shown in (a), the parameter estimate of $a_{2}$ is shown in (b), the parameter estimate of $a_{3}$ is shown in (c), the parameter estimate of $b_{1}$ is shown in (d), the parameter estimate of $b_{2}$ is shown in (e) and the parameter estimate of $b_{3}$ is shown in (f). Moreover, the mark " $\circ$ " denotes the original value, the mark " $*$ " denotes the estimated value obtained by the recursive gradient (RG) method without separation and the mark " $\triangle$ " denotes the estimated value obtained by the separable recursive gradient (SRG) method. 
Table 1. The SRG estimates and their estimation errors by the 20 Monte Carlo tests

\begin{tabular}{cccccc}
\hline$k$ & 200 & 500 & 1000 & 1500 & True values \\
\hline$a_{1}$ & $5.19738 \pm 0.20447$ & $5.21363 \pm 0.18877$ & $5.24370 \pm 0.16165$ & $5.27319 \pm 0.13560$ & 5.40000 \\
$a_{2}$ & $4.01297 \pm 0.29542$ & $4.07954 \pm 0.23498$ & $4.20557 \pm 0.12641$ & $4.33246 \pm 0.06724$ & 4.30000 \\
$a_{3}$ & $3.00986 \pm 0.19044$ & $3.01192 \pm 0.18882$ & $3.01595 \pm 0.18559$ & $3.02015 \pm 0.18178$ & 3.20000 \\
$b_{1}$ & $0.98662 \pm 0.07630$ & $1.04011 \pm 0.09422$ & $1.07623 \pm 0.20569$ & $1.03866 \pm 0.20443$ & 1.00000 \\
$b_{2}$ & $2.02968 \pm 0.11315$ & $2.09499 \pm 0.15179$ & $2.14158 \pm 0.25357$ & $2.10367 \pm 0.26399$ & 2.00000 \\
$b_{3}$ & $1.87035 \pm 0.21571$ & $1.86617 \pm 0.30278$ & $1.87574 \pm 0.35708$ & $1.91011 \pm 0.39457$ & 1.80000 \\
\hline
\end{tabular}

Table 2. The SRG and the RG estimates and their estimation errors ( $\mathrm{SNR}=1.04)$

\begin{tabular}{ccccccccr}
\hline Method & $k$ & $a_{1}$ & $a_{2}$ & $a_{3}$ & $b_{1}$ & $b_{2}$ & $b_{3}$ & $\delta(\%)$ \\
\hline SRG & 100 & 5.18814 & 3.97352 & 3.00857 & 0.83499 & 1.70324 & 1.26882 & 9.41002 \\
& 200 & 5.19229 & 3.99105 & 3.00913 & 0.88594 & 1.80900 & 1.45368 & 7.21713 \\
& 500 & 5.20472 & 4.04362 & 3.01084 & 0.89871 & 1.83158 & 1.48244 & 6.49668 \\
& 1000 & 5.22544 & 4.13125 & 3.01367 & 0.90558 & 1.83896 & 1.47662 & 5.93689 \\
& 2000 & 5.26688 & 4.30651 & 3.01934 & 0.91899 & 1.85333 & 1.46500 & 5.37002 \\
\hline RG & 100 & 5.09758 & 3.59083 & 2.99620 & 0.78662 & 1.60216 & 1.09031 & 14.25280 \\
& 200 & 5.08594 & 3.54165 & 2.99461 & 0.82521 & 1.68325 & 1.23474 & 13.28037 \\
& 500 & 5.08416 & 3.53414 & 2.99437 & 0.83110 & 1.69562 & 1.25677 & 13.15755 \\
& 1000 & 5.08416 & 3.53413 & 2.99436 & 0.83111 & 1.69565 & 1.25682 & 13.15727 \\
& 2000 & 5.08416 & 3.53413 & 2.99436 & 0.83111 & 1.69565 & 1.25682 & 13.15727 \\
\hline \multicolumn{2}{c}{ True values } & 5.40000 & 4.30000 & 3.20000 & 1.00000 & 2.00000 & 1.80000 & \\
\hline
\end{tabular}

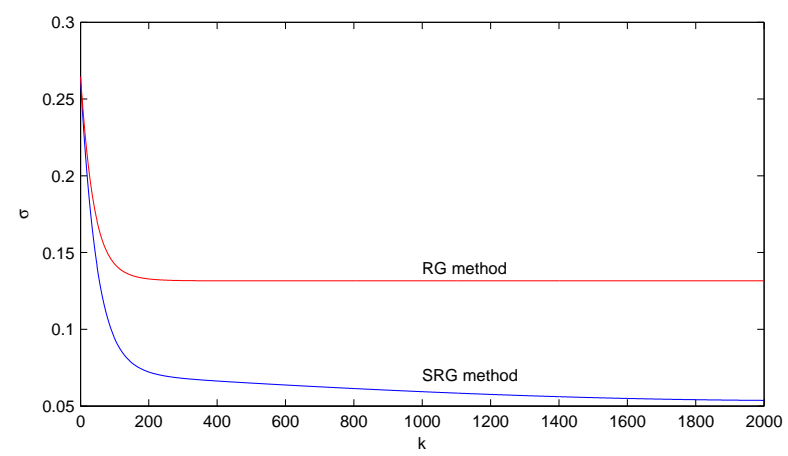

Fig. 2. The SRG and RG estimation errors versus $k$ $(\mathrm{SNR}=1.04)$

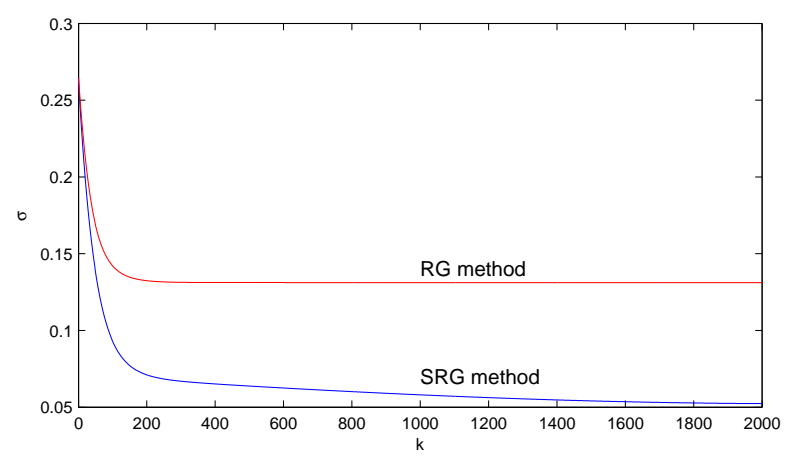

Fig. 3. The SRG and RG estimated errors versus $k$ $(\mathrm{SNR}=26.17)$
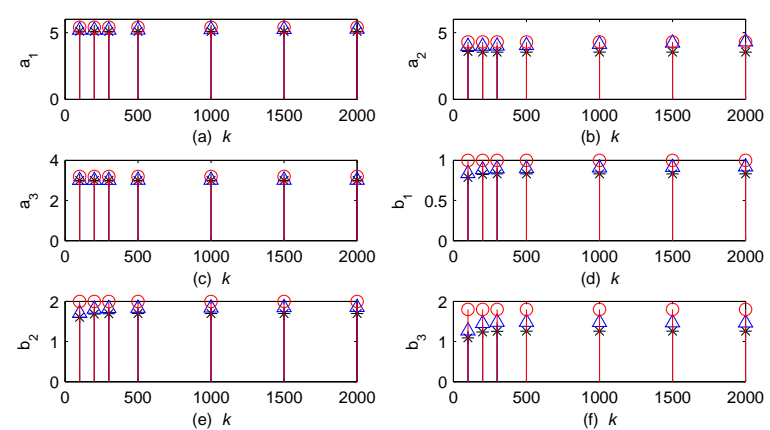

Fig. 4. The SRG and RG estimated parameters $(\mathrm{SNR}=1.04)$

In Figure 5, the parameter estimate of $a_{1}$ is shown in (a), the parameter estimate of $a_{2}$ is shown in (b), the parameter estimate of $a_{3}$ is shown in (c), the parameter estimate of $b_{1}$ is shown in (d), the parameter estimate of $b_{2}$ is shown in (e) and the parameter estimate of $b_{3}$ is shown in (f). Moreover, the mark " $\circ$ " denotes the original value, the mark " $*$ " denotes the estimated value obtained by the recursive gradient (RG) method without separation and the mark " $\triangle$ " denotes the estimated value obtained by the separable recursive gradient (SRG) method. 
Table 3. The SRG and the RG estimates and their estimation errors (SNR=26.17)

\begin{tabular}{|c|c|c|c|c|c|c|c|c|}
\hline Method & $k$ & $a_{1}$ & $a_{2}$ & $a_{3}$ & $b_{1}$ & $b_{2}$ & $b_{3}$ & $\delta(\%)$ \\
\hline \multirow[t]{5}{*}{ SRG } & 100 & 5.18818 & 3.97365 & 3.00857 & 0.83762 & 1.70867 & 1.27815 & 9.29701 \\
\hline & 200 & 5.19235 & 3.99131 & 3.00914 & 0.88905 & 1.81536 & 1.46431 & 7.10602 \\
\hline & 500 & 5.20488 & 4.04428 & 3.01086 & 0.90222 & 1.83850 & 1.49298 & 6.37959 \\
\hline & 1000 & 5.22575 & 4.13257 & 3.01371 & 0.90963 & 1.84659 & 1.48662 & 5.80708 \\
\hline & 2000 & 5.26751 & 4.30914 & 3.01943 & 0.92410 & 1.86234 & 1.47395 & 5.23553 \\
\hline \multirow[t]{5}{*}{$\overline{\mathrm{RG}}$} & 100 & 5.09708 & 3.58877 & 2.99613 & 0.78827 & 1.60561 & 1.09636 & 14.20637 \\
\hline & 200 & 5.08537 & 3.53931 & 2.99454 & 0.82709 & 1.68716 & 1.24159 & 13.24110 \\
\hline & 500 & 5.08359 & 3.53177 & 2.99429 & 0.83301 & 1.69960 & 1.26375 & 13.11990 \\
\hline & 1000 & 5.08359 & 3.53175 & 2.99429 & 0.83302 & 1.69963 & 1.26380 & 13.11962 \\
\hline & 2000 & 5.08359 & 3.53175 & 2.99429 & 0.83302 & 1.69963 & 1.26380 & 13.11962 \\
\hline \multicolumn{2}{|c|}{ True values } & 5.40000 & 4.30000 & 3.20000 & 1.00000 & 2.00000 & 1.80000 & \\
\hline
\end{tabular}
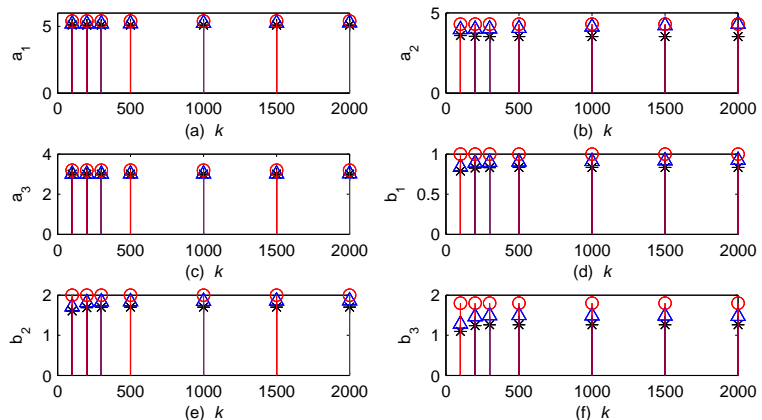

Fig. 5. The SRG and RG estimated parameters $(\mathrm{SNR}=26.17)$

\section{CONCLUSIONS}

This paper studies the the identification of the transfer functions of process control systems for identifying the industrial process. For the purpose of obtaining higher estimation accuracy and on-line identification, a strategy of using dynamical data with increasing length is developed and applied to derive the identification method based on system response signals. Because the system response function is highly nonlinear, we analyze the relation between the system response function and the system parameters to be identified, and design a separable identification algorithm by separating the whole parameters of the systems into two parameter vectors. On the basis of the separated parameter vectors and the gradient optimization, two separated identification models are built and two sub-algorithms are derived by combining them together and reciprocal estimation. Finally, the separable recursive gradient method is proposed for the process control systems. The numerical simulation results concerning the performance test and comparison show that the proposed separable recursive gradient method is practically feasible, which can be adopted for industrial applications. The proposed separable recursive gradient algorithm for dynamical systems based on the impulse response signals can combine and other estimation methods [57-59] to explore new identification methods and can be applied to other fields [60-64] such as information processing and engi- neering systems [65-69] and so on.

\section{REFERENCES}

[1] M. Gan, C.L.P. Chen, G.Y. Chen, and L. Chen, "On some separated algorithms for separable nonlinear squares problems," IEEE Transactions on Cybernetics, vol. 48, no. 10, pp. 2866-2874, October 2018.

[2] L. Xu, F. Ding, "Parameter estimation for control systems based on impulse responses," International Journal of Control, Automation, and Systems, vol. 15 , no. 6, pp. 2471-2479, 2017.

[3] G.Y. Chen, M. Gan, C.L.P. Chen, and H.X. Li, "A regularized variable projection algorithm for separable nonlinear least-squares problems," IEEE Transactions on Automatic Control, vol. 64, no. 2, pp. 526-537, February 2019.

[4] H. Ma, J. Pan, F. Ding, L. Xu, and W. Ding, "Partially-coupled least squares based iterative parameter estimation for multi-variable output-error-like autoregressive moving average systems," IET Control Theory and Applications, vol. 13, no. 18, pp. 3040-3051, December 2019.

[5] J. Ding, J.Z. Chen, J.X. Lin, and L.J. Wan, "Particle filtering based parameter estimation for systems with output-error type model structures," Journal of the Franklin Institute, vol. 356, no. 10, pp. 5521-5540, July 2019.

[6] M.H. Li and X.M. Liu, "The least squares based iterative algorithms for parameter estimation of a bilinear system with autoregressive noise using the data filtering technique," Signal Processing, vol. 147, pp. 23-34, June 2018.

[7] J.X. Ma, W.L. Xiong, and J. Chen, "Hierarchical identification for multivariate Hammerstein systems by using the modified Kalman filter," IET Control Theory and Applications, vol. 11, no. 6, pp. 857-869, April 2017.

[8] Y. Gu, Y. Chou, J. Liu, and Y. Ji, "Moving horizon estimation for multirate systems with time-varying time-delays," Journal of the Franklin Institute, vol. 356, no. 4, pp. 2325-2345, March 2019.

[9] M. Gan, H.X. Li, H. Peng, "A variable projection approach for efficient estimation of RBF-ARX model," IEEE Transactions on Cybernetics, vol. 45, no. 3, pp. 476-485, March 2015.

[10] L.J. Wang, Y. Ji, L.J. Wan, and N. Bu, "Hierarchical recursive generalized extended least squares estimation algorithms for a class of nonlinear stochastic systems with colored noise," Journal of the Franklin Institute, vol. 356, no. 16, pp. 10102-10122, November 2019.

[11] J.X. Ma and F. Ding, "Filtering-based multistage recursive identification algorithm for an input nonlinear output-error autoregressive system by using key the term separation technique,"Circuits Systems and Signal Processing, vol. 36, no. 2, pp. 577-599, February 2017.

[12] F. Ding, F.F. Wang, L. Xu, and M.H. Wu, "Decomposition based least squares iterative identification algorithm for multivariate pseudo-linear ARMA systems using the data filtering," Journal of the Franklin Institute, vol. 354, no. 3, pp. 1321-1339, February 2017.

[13] C.Q. Guo, L.J. Wang, and F. Deng, "The auxiliary model based hierarchical estimation algorithms for bilinear stochastic systems with colored noises," International Journal of Control Automation and Systems, vol. 18, no. 3, pp. 650-660, March 2020.

[14] M.H. Li, X.M. Liu, and F. Ding, "The filtering-based maximum likelihood iterative estimation algorithms for a special class of nonlinear systems with autoregressive moving average noise using the hierarchical identification principle,"International Journal of Adaptive Control and Signal Processing, vol. 33, no. 7, pp. 1189-1211, July 2019.

[15] X.K. Wan, Y. Li, C. Xia, M.H. Wu, J. Liang, and N. Wang, "A T-wave alternans assessment method based on least squares curve fitting technique," Measurement, vol. 86, pp. 93-100, May 2016.

[16] J. Pan, W. Li, and H.P. Zhang, "Control algorithms of magnetic suspension systems based on the improved double exponential reaching law of sliding mode control," International Journal of Control Automation and Systems, vol. 16, No. 6, pp. 2878-2887, December 2018. 
[17] M.Y. Liu, H.F. Ku, J.X. Zhang, P.D. Xu, and C.H. Wu, "Predicting fatigue life for finite line contact under starved elastohydrodynamic lubrication condition," Mathematical Problems in Engineering, vol. 2020, p. 5928621, January 2020.

[18] M.Y. Liu, P.D. Xu, J.X. Zhang, and H. Ding, "Analysis of mechanical power loss of a helical gear pair based on the starved thermal-elastohydrodynamic lubrication model," Industrial Lubrication and Tribology, vol. 72, 2020. doi:10.1108/ILT-06-2019-0225

[19] M. Gan, C.L. Philip Chen, G.Y. Chen, L. Chen, "On some separated algorithm for separable nonlinear least squares problems," IEEE Transaction on Cybernetics, vol. 48, no. 10, pp. 2866-2874, 2018.

[20] Y.J. Wang, F. Ding, and M.H. Wu, "Recursive parameter estimation algorithm for multivariate output-error systems," Journal of the Franklin Institute, vol. 355, no. 12, pp. 5163-5181, August 2018.

[21] P. Ma and F. Ding, "New gradient based identification methods for multivariate pseudo-linear systems using the multi-innovation and the data filtering," Journal of the Franklin Institute, vol. 354, no. 3, pp. 1568-1583, February 2017.

[22] C.P. Yu, L. Ljung, A. Wills, and M. Verhaegen, "Constrained subspace method for the identification of structured state-space models," IEEE Transactions on Automatic Control, vol. 65, 2020 doi:10.1109/TAC.2019.2957703

[23] J. Pan, X. Jiang, X.K. Wan, W. Ding, "A filtering based multi-innovation extended stochastic gradient algorithm for multivariable control systems," International Journal of Control Automation and Systems, vol. 15, no. 3 , pp. 1189-1197, 2017.

[24] J. Ding, J.Z. Chen, J.X. Lin, and G.P. Jiang, "Particle filtering-based recursive identification for controlled auto-regressive systems with quantised output," IET Control Theory and Applications, vol. 13, no. 14, pp. 2181-2187, September 2019

[25] L.J. Wan and F. Ding, "Decomposition- and gradient-based iterative identification algorithms for multivariable systems using the multi-innovation theory," Circuits Systems and Signal Processing, vol. 38, no. 7, pp. 2971-2991, July 2019.

[26] Y. Cao, Z. Wang, F. Liu, P. Li, and G. Xie, "Bio-inspired speed curve optimization and sliding mode tracking control for subway trains," IEEE Transactions on Vehicular Technology, vol. 68, no. 7, pp. 6331-6342, July 2019.

[27] Y. Cao, Y.K. Sun, G. Xie, and T. Wen, "Fault diagnosis of train plug door based on a hybrid criterion for IMFs selection and fractional wavelet package energy entropy," IEEE Transactions on Vehicular Technology, vol. 68 , no. 8, pp. 7544-7551, August 2019 .

[28] S.Y. Liu, F. Ding, L, Xu, T. Hayat, "Hierarchical principle-based iterative parameter estimation algorithm for dual-frequency signals," Circuits Systems and Signal Processing, vol. 38, no. 7, pp. 3251-3268, July 2019.

[29] Y. Cao, H. Lu, and T. Wen, A safety computer system based on multi-sensor data processing," Sensors, vol. 19, no. 4, p. 818, February 2019.

[30] Y. Cao, Y. Zhang, T. Wen, and P. Li, "Research on dynamic nonlinear input prediction of fault diagnosis based on fractional differential operator equation in high-speed train control system," Chaos, vol. 29, no. 1, p. 013130, January 2019.

[31] L.J. Liu, F. Ding, L. Xu, J. Pan, A. Alsaedi, and T. Hayat, "Maximum likelihood recursive identification for the multivariate equation-error autoregressive moving average systems using the data filtering," IEEE Access, vol. 7, pp. 41154-41163, 2019.

[32] C.C. Yin and C.W. Wang, "The perturbed compound Poisson risk process with investment and debit interest," Methodology and Computing in Applied Probability, vol. 12, no. 3, pp. 391-413, September 2010.

[33] C.C. Yin and K.C. Yuen, "Optimality of the threshold dividend strategy for the compound Poisson model," Statistics \& Probability Letters, vol. 81, no. 12, pp. 1841-1846, December 2011.

[34] C.C. Yin and Y.Z. Wen, "Optimal dividend problem with a terminal value for spectrally positive Levy processes," Insurance Mathematics \& Economics, vol. 53, no. 3, pp. 769-773, November 2013

[35] C.C. Yin and Y.Z. Wen, "Exit problems for jump processes with applications to dividend problems," Journal of Computational and Applied Mathematics, vol. 245, pp. 30-52, June 2013.

[36] F. Yang, J.L. Fu, and X.X. Li, "A potential-free field inverse Schröinger problem: optimal error bound analysis and regularization method," Inverse Problems in Science and Engineering, vol. 28, 2020. https://doi.org/10.1080/17415977.2019.1700243

[37] F. Yang, N. Wang, and X.X. Li, "Landweber iterative method for an inverse source problem of time-fractional diffusion-wave equation on spherically symmetric domain," Journal of Applied Analysis and Computation, vol. 10 no. 2, pp. 1-16, April 2020.

[38] C.C. Yin and Y.Z. Wen, "An extension of Paulsen-Gjessing's risk model with stochastic return on investments," Insurance Mathematics \& Economics, vol. 52, no. 3, pp. 469-476, May 2013.

[39] C.C. Yin, Y.Z. Wen, and Y.X. Zhao, "On the optimal dividend problem for a spectrally positive levy process," Astin Bulletin, vol. 44, no. 3, pp. 635-651, September 2014
[40] C.C. Yin and K.C. Yuen, "Exact joint laws associated with spectrally negative Levy processes and applications to insurance risk theory," Frontiers of Mathematics in China, vol. 9, no. 6, pp. 1453-1471, December 2014

[41] C.C. Yin and K.C. Yuen, 'Optimal dividend problems for a jump-diffusion model with capital injections and proportional transaction costs," Journal of Industrial and Management Optimization, vol. 11, no. 4, pp. 1247-1262, October 2015

[42] S.S. Tian, X.X. Zhang, S. Xiao, J. Zhang, Q. Chen, and Y. Li, "Application of $\mathrm{C} 6 \mathrm{~F} 12 \mathrm{O} / \mathrm{CO} 2$ mixture in $10 \mathrm{kV}$ medium-voltage switchgear," IET Science Measurement \& Technology, vol. 13, no. 9, pp. 1225-1230, November 2019.

[43] G.Z. Zhang, X.X. Zhang, H.T. Cheng, and J. Tang, "Ladder-Wise calculation method for Z-coordinate of transformer PD source based on planar layout UHF antenna sensors," IEEJ Transactions on Electrical and Electronic Engineering, vol. 15, no. 3, pp. 340-345, March 2020.

[44] Y. Zhang, X.X. Zhang, Y. Li, Y.L. Li, Q. Chen, G.Z. Zhang, S. Xiao, J. Tang, "AC breakdown and decomposition characteristics of environmental friendly gas C5F10O/Air and C5F10O/N-2," IEEE Access, vol. 7, pp. 73954-73960, 2019

[45] Z.W. Chen, X.X. Zhang, H. Xiong, D.C. Chen, H.T. Cheng, J. Tang, Y Tian, S. Xiao, "Dissolved gas analysis in transformer oil using Pt-doped WSe2 monolayer based on first principles method," IEEE Access, vol. 7, pp. 72012-72019, 2019.

[46] X. Zhang and F. Ding, "Hierarchical parameter and state estimation for bilinear systems," International Journal of Systems Science, vol. 51, no. 2, pp. 275-290, 2020.

[47] P.C. Gong, W.Q. Wang, X.R. Wan, "Adaptive weight matrix design and parameter estimation via sparse modeling for MIMO radar," Signal Processing, vol. 139, pp. 1-11, October 2017.

[48] P.C. Gong, W.Q. Wang, F.C. Li, and H. Cheung, "Sparsity-aware transmi beamspace design for FDA-MIMO radar," Signal Processing, vol. 144, pp. 99-103, March 2018.

[49] N. Zhao, "Joint optimization of Cooperative Spectrum Sensing and Resource Allocation in Multi-channel Cognitive Radio Sensor Networks," Circuits Systems and Signal Processing, vol. 35, no. 7, pp. 2563-2583, July 2016.

[50] N. Zhao, M.H. Wu, and J.J. Chen, "Android-based mobile educational platform for speech signal processing," International Journal of Electrical Engineering Education, vol. 54, no. 1, pp. 3-16, January 2017.

[51] N. Zhao, Y. Liang, and Y. Pei, "Dynamic contract incentive mechanism for cooperative wireless networks," IEEE Transactions on Vehicular Technology, vol. 67, no. 11, pp. 10970-10982, November 2018.

[52] X.L. Zhao, F. Liu, B. Fu, and F. Na, "Reliability analysis of hybrid multicarrier energy systems based on entropy-based Markov model," Proceeding of the Institution of Mechanical Engineers Part O-Journal of Risk and Reliability, vol. 230, no. 6, pp. 561-569, December 2016.

[53] X.L. Zhao, Z.Y. Lin, B. Fu, L. He, and F. Na, "Research on automatic generation control with wind power participation based on predictive optimal 2-degree-of-freedom PID strategy for multi-area interconnected power system," Energies, vol. 11, no. 12, Article Number: 3325, December 2018. http://dx.doi.org/10.3390/en11123325

[54] L. Wang, H. Liu, L.V. Dai, and Y.W. Liu, "Novel method for identifying fault location of mixed lines," Energies, vol. 11, no. 6, Article Number: 1529, June 2018. https://doi.org/10.3390/en11061529

[55] H. Liu, Q.X. Zou, Z.P. Zhang, "Energy disaggregation of appliances consumptions using ham approach," IEEE Access, vol. 7, pp. 185977-185990, 2019.

[56] X.S. Zhan, L.L. Cheng, J. Wu, and H.C. Yan, "Modified tracking performance limitation of networked time-delay systems with two-channel constraints," Journal of the Franklin Institute, vol. 356, no. 12, pp. 6401-6418, August 2019.

[57] F. Ding, L. Xu, D.D. Meng, X.B. Jin, A. Alsaedi, T. Hayat, "Gradient estimation algorithms for the parameter identification of bilinear systems using the auxiliary model," Journal of Computational and Applied Mathematics, vol. 369, p. 112575, May 2020.

[58] X. Zhang, F. Ding, and E.F. Yang, "State estimation for bilinear systems through minimizing the covariance matrix of the state estimation errors," International Journal of Adaptive Control and Signal Processing, vol. 33 , no. 7, pp. 1157-1173, July 2019.

[59] F. Ding, L. Lv, J. Pan, X.K. Wan, and X.B. Jin,"Two-stage gradient-based iterative estimation methods for controlled autoregressive systems using the measurement data," International Journal of Control Automation and Systems, vol. 18, 2020. http://dx.doi.org/10.1007/s12555-019-0140-3.

[60] W. X. Shi, N. Liu, Y. M. Zhou, X. A. Cao, "Effects of postannealing on the characteristics and reliability of polyfluorene organic light-emitting diodes," IEEE Transactions on Electron Devices, vol. 66, no. 2, pp. 1057-1062, February 2019.

[61] N. Liu, S. Mei, D. Sun, W. Shi, J. Feng, Y.M. Zhou, F. Mei, J. Xu, Y. Jiang, and X.A. Cao, "Effects of charge transport materials on blue fluorescent organic light-emitting diodes with a host-dopant system," Micromachines, vol. 10, no. 5, Article Number: 344, May 2019 https://doi.org/10.3390/mi10050344 
[62] T. Z. Wu, X. Shi, L. Liao, C. J. Zhou, H. Zhou, Y. H. Su, "A capacity configuration control strategy to alleviate power fluctuation of hybrid energy storage system based on improved particle swarm optimization," Energies, vol. 12, no. 4, Article Number: 642, February 2019. https://doi.org/10.3390/en12040642

[63] X.L. Zhao, Z.Y. Lin, B. Fu, L. He, C.S. Li, "Research on the predictive optimal PID plus second order derivative method for AGC of power system with high penetration of photovoltaic and wind power," Journal of Electrical Engineering \& Technology, vol. 14, no. 3, pp. 1075-1086, May 2019.

[64] N. Li, S. Guo, and Y. Wang, "Weighted preliminary-summation-based principal component analysis for non-Gaussian processes," Control Engineering Practice, vol. 87, pp. 122-132, June 2019.

[65] G.C. Yang, Z.J. Chen, Y. Li, and Z.D. Su, "Rapid relocation method for mobile robot based on improved ORB-SLAM2 algorithm," Remote Sensing, vol. 11, no. 2, Article Number: 149, January 2019. doi:10.3390/rs11020149

[66] Z.D. Su, Y. Li, and G.C. Yang, "Dietary composition perception algorithm using social robot audition for Mandarin Chinese," IEEE Access, vol. 8, 2020. doi:10.1109/ACCESS.2019.2963560

[67] Y.F. Chang, G.S. Zhai, B. Fu, L.L. Xiong, "Quadratic stabilization of switched uncertain linear systems: a convex combination approach," IEEECAA Journal of Automatica Sinica, vol. 6, no. 5, pp. 1116-1126, September 2019.

[68] L. He, H. Lin, Q. Zou, D.J. Zhang, "Accurate measurement of pavement deflection velocity under dynamic loads," Automation in Construction, vol. 83, pp. 149-162, November 2017.

[69] J.G. Zhou, H.L. Xiao, W.W. Jiang, W.F. Bai, and G.L. Liu, "Automatic subway tunnel displacement monitoring using robotic total station," Measurement, vol. 151, p. 107251, February 2020.

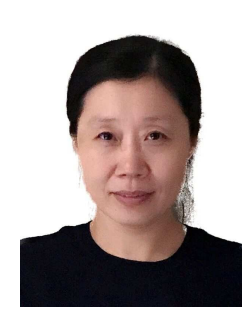

Ling Xu was born in Tianjin, China. She received the Master and Ph.D. degrees from the Jiangnan University (Wuxi, China), in 2005 and 2015, respectively. She is a Post-Doctoral Fellow at the Jiangnan University and has been an Associate Professor since 2015. She is a Colleges and Universities "Blue Project" Young Teacher (Jiangsu, China). Her research interests include process control, parameter estimation and signal modeling.

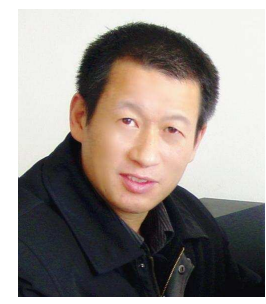

Feng Ding received his B.Sc. degree from the Hubei University of Technology (Wuhan, China) in 1984, and his M.Sc. and $\mathrm{Ph} . \mathrm{D}$. degrees both from the Tsinghua University, in 1991 and 1994, respectively.

He has been a professor in the School of Internet of Things Engineering at the Jiangnan University (Wuxi, China) since 2004. His current research interests include model identification and adaptive control. He authored four books on System Identification.

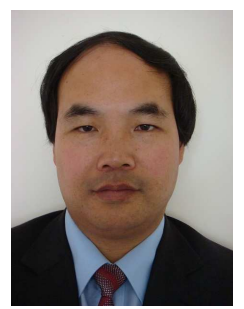

Erfu Yang received the B.Eng. and M.Eng. degrees both in Aerospace Propulsion Theory and Engineering from Beijing University of Aeronautics and Astronautics (Beijing, China) and the Ph.D. degree in Robotics from the School of Computer Science and Electronic Engineering at the University of Essex (Colchester, UK) in 2008. He is currently a Lecturer in the Department of Design, Manufacturing and Engineering Management (DMEM), the University of Strathclyde, Glasgow, UK. His main research interests include robotics, mechatronics, machine learning and artificial intelligence, etc. He has published more than 55 journal papers and 10 book chapters.

Dr. Yang has been awarded over 15 research grants as PI (principal investigator) or CI (co-investigator). He is the Fellow of the UK Higher Education Academy, member of IEEE and IEEE Society of Robotics and Automation, committee member of the Chinese Automation and Computing Society in the UK (CACSUK), and the IET SCOTLAND Manufacturing Technical Network. He is also an associate editor for the Cognitive Computation journal published by Springer. 